\title{
The mechanism and clinical effectiveness of the six-step Chinese Daoyin technique on hemiplegic patients with drop-foot ${ }^{*}$
}

\author{
Wen-Ru Zhao , Hai-Hong Zhao, Xue-Min Zhang, Xiao Cao, Xin Li \\ Neurological Training Rehabilitation Department, Rehabilitation Hospital of National Research Center for Rehabilitation Technical \\ Aids (NRRA), Beijing, China \\ Email: "zhaowenru@hotmail.com
}

Received 25 August 2012; revised 27 September 2012; accepted 5 October 2012

\begin{abstract}
Objective: The aim of this study was to improve the Chinese Daoyin technique (physical and breathing exercises), which is an ancient rehabilitation method, to elevate its effect and to standardize its operational procedure. Methods: Based on the ancient Chinese Daoyin technique, a six-step method was developed including: 1) motion imagination and imitation; 2) mental and breathing regulation; 3 ) target point determination; 4) motion percussion; 5) motion persistence; 6) relaxation. Sixty drop-foot patients who suffered from hemiparesis after a stroke were randomly divided into the experimental group and the control group. Functional evaluation including Fugl-Meyer assessment score, Lovett manual muscle test (MMT), active range of motion of the ankle joint and electromyography were performed before and after the training course. The data were analyzed using the statistical software SPSS 12.0. Results: The differences between the two groups were significant. The effectiveness of the six-step group was significantly better than the effectiveness of the control group. Conclusion: The six-step method of the Chinese Daoyin technique is an effective rehabilitation training method for drop-foot in stroke patients with hemiparesis.
\end{abstract}

Keywords: Rehabilitation; Chinese Daoyin Technique; Six-Step Method; Hemiparesis; Drop-Foot

\section{INTRODUCTION}

The treatment of motor function loss due to brain damage such as cerebral embolism, cerebral infarction, cerebral hemorrhage, and cerebral trauma must focus on the

\footnotetext{
*Announcement: This paper has been published in Chinese in the Chinese Journal of Rehabilitation Medicine.

${ }^{\#}$ Corresponding author.
}

restoration of brain function. Traditional rehabilitation techniques such as Bobath, Rood, Brunnstrom, proprioceptive neuromuscular facilitation (PNF) and electrical stimulation mainly consist of passive movement and afferent stimulation, using primitive reflexes such as the stretch reflex and the tonic neck reflex to induce spontaneous movement of the body. These methods mainly use the lower central nervous system. It is comparatively less effective for the restoration of brain function, and results in lower treatment effectiveness, especially in patients who have been affected for more than six months.

According to the theory that the plasticity of the central nervous system (CNS) can only be promoted by technical application of body movement, the Chinese Daoyin technique is a very important method for motor function recovery after CNS damage, because it can facilitate brain plasticity through its core axiom, which is "before performing an initiative movement of the body, one needs to first adjust the mind, then adjust the breathing”. This technique is over 2000 years old and was founded in the period of Chun Qiu and the Warring States (before the Tang Dynasty). A famous ancient Chinese medicine book published during the Sui Dynasty (610 A.D.), called the "General Treatise on the Causes and Symptoms of Diseases" [1] enumerated more than twenty types of skills of the Daoyin technique for rehabilitation of hemiparesis. The common ground of these skills is adjusting the mind first and then adjusting breathing while being guided by the mind, and eventually performing an initial movement. The main effect of this technique is to dredge the meridian channels, regulate "qi (energy of the body) and blood", coordinate the viscera, strengthen healthy qi and finally eliminate pathogenic factors from the body. It can enhance the homeostatic mechanism of the body. Therefore, the Daoyin technique can influence the metabolic processes of the entire body. It has been demonstrated that the Daoyin technique has good adjustive and rehabilitative 
effects on cardiovascular, respiratory, neurological and gastrointestinal diseases [2]. In the past generations, Chinese medicine specialists have supplemented and developed many aspects of this technique based on the experiences of their predecessors. Many of these developments are worth studying and passing on [3].

Unfortunately, from the very beginning of the development of this technique, it has had an inseparable relationship with the traditional Chinese culture. During its continual developmental process, it has been supplemented, modified and revised by each denomination. This made the Daoyin technique appear very extensive and profound in its theoretical contents, but it was inevitably surrounded with mystery. Because of the above reason, and the fact that the effectiveness and mechanism of the Daoyin technique has never been confirmed by research, it includes unclear concepts, and subjective, abstract principles. Moreover, the Daoyin technique consisted mainly of whole-body movements such as "celestial being showing the way" (Figure 1), and imitation of animal motions, which are quite difficult to do for hemiplegic patients, especially in severe cases. Therefore, since the Qing Dynasty, the Daoyin technique has been mainly used for health cultivation and health maintenance, but has rarely been applied to clinical rehabilitation. In other words, it is very close to extinction.

According to the theories that "active motion can develop CNS potency" and "rehabilitation exercise is more efficient on a "step by step" and "from easy to difficult" basis, we have improved the Daoyin technique to a sixstep method while continuing its basic Chinese medicine mechanisms. It has been confirmed as an effective method for restoring functional movement after CNS damage.

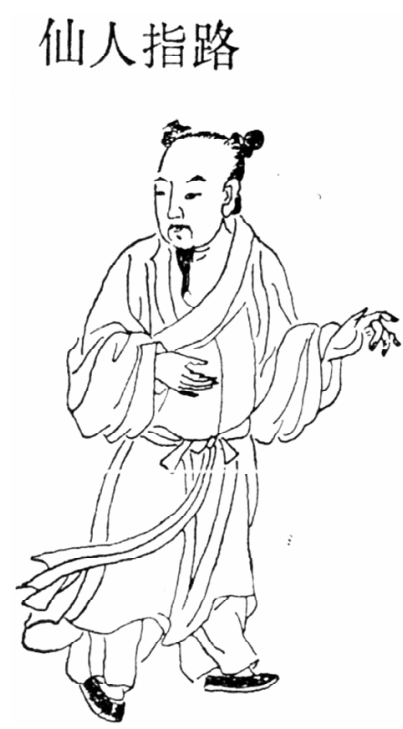

Figure 1. Celestial being showing the way.

\section{MATERIALS AND METHODS}

\subsection{Improving the Chinese Daoyin Technique}

Using the theories of motor program formation in the motor center of the cerebral cortex, the potential exploitation of the human body, and the meridian theories of traditional Chinese medicine [4-6], a six-step method was developed based on the ancient Chinese Daoyin technique. The steps are as follows:

1) Motion imagination and imitation: Explain to the patient that the brain controls body movements by releasing electrical signals. First, daoyin the patient to perform a single joint motion (e.g., dorsiflexion) on the nonhemiparetic side, then daoyin the patient to imitate and perform the same motion using the affected side. Tell the patient to imagine the normal motion of the hemiparetic side to help the patient establish movement intention and the goal of execution.

2) Mental and breathing regulation: Guide the patient to relax in a quiet room, enter a state of meditation, and gather all the energy of the body to the injured side of the brain, entering a condition in which the patient is ready to perform a motion.

3) Target point determination: Inform the patient of the target muscle on which energy should be released (e.g., the anterior tibial muscle is the target muscle for foot dorsiflexion).

4) Motion percussion: Daoyin the patient to take a deep breath, then suddenly release all of the gathered energy to the target muscle and produce the joint motion (e.g., foot dorsiflexion), which results from the muscle contraction. This step can centralize all of the energy to a single location to achieve better results.

5) Persistence: The physical therapist applies an adequate amount of resistance against the direction of the joint motion (e.g., when in foot dorsiflexion, resistance should be applied on the dorsum of the foot at the point of the metatarsophalangeal joints, even when the anterior tibial muscle strength is zero). This will help the patient's brain to find the direction in which the brain electrical signals should be released. When joint motion reaches its full extent, the therapist encourages the patient to maintain it for 6 seconds by counting from 1 to 6 . During this time, the therapist encourages the patients by using phrases such as "keep it up or you'll fall!", "higher or you'll fall!" to create a "critical (dangerous and urgent) condition", to enhance potential exploitation of the brain. When performing foot dorsiflexion, daoyin the patient to increase only the signals released to the anterior tibial muscle, while simultaneously paying attention to the decrease of signals released to the gastrocnemius muscle. This will enhance motor coordination.

6) Relaxation: When finished with the motion, daoyin the patient to inhale deeply but exhale slowly (inhalation 
time: exhalation time $=1: 2$ ), relaxing the whole body and soul as much as possible, this will dredge the meridian channels and dismiss fatigue.

When one set of the six-step Daoyin technique is completed, the physical therapist can perform passive joint motion and muscle massage, which can loosen the adhesion of articulations and facilitate blood circulation of the muscle. Then, we can proceed to the next set of the six-step Daoyin technique.

\subsection{Clinical Application}

1) Subjects: 60 patients with drop-foot secondary to stroke were randomly selected for this study. All subjects were inpatients at The Neurological Training Rehabilitation Center of the Beijing Tongren Hospital from June 2006 to June 2008, and had a case history of 1 to 5 years. They were randomly and evenly divided into the ex- perimental and control groups.

2) Group division

a) Experimental group: The experimental group consisted of 18 male subjects and 12 female subjects, their ages ranged from 35 to 76 years, the average age was 48.4 years. Case history time ranged from 1 year and 7 months to 4 years and 11 months, the average was 3 years and 4 months.

b) Control group: The control group consisted of 16 male subjects and 14 female subjects, their ages ranged from 34 to 71 years and the average age was 47.8 years. Case history time ranged from one year to five years and the average time was three years and six months.

3) Treatment methods

Experimental group: The patients were trained to perform active foot dorsiflexion using the six-step Daoyin technique as described above in detail. The training time was 45 minutes per session, one session per day, and 30 sessions per course in total.

Control group: Patients were treated with the Brunnstrom foot dorsiflexion training method [7], which included the following:

a) Bechterew/Marie Foix reflex: The physical therapist pushes the patient's toes to passive plantar flexion, which stretches the muscle spindle of the dorsiflexor of the toes, causing a reflex that the results in dorsiflexion of the toes and the foot as well as knee and hip flexion. The time for this session was 15 minutes.

b) Percussion: The therapist percusses the lateral side of the dorsum pedis rapidly with his/her fingertips to induce dorsiflexion. The time for this session was 15 minutes.

c) Stimulation by skin brushing: The therapist uses a brush to stimulate the lateral side of the dorsum pedis for 30 seconds to induce foot dorsiflexion. The time for this session was 15 minutes.
The above three treatment methods took 45 minutes, once a day and 30 sessions in total.

4) Assessment of function

Evaluation methods included Lovett MMT of the anterior tibial muscle, active range of motion (AROM) of the ankle joint, Fugl-Meyer assessment score of foot dorsiflexion ( 0 points: no active dorsiflexion; 1 point: partial active dorsiflexion; 2 points: full range active dorsiflexion), and electromyography (EMG) of the anterior tibial muscle (we used the tetra-lead Neuroeducator III manufactured by Therapeutic Alliances, USA; and Ag-AgCl surface electrodes manufactured by $3 \mathrm{M}$ ). Rehabilitation assessment was performed twice, once before and once after completion of the entire course of treatment.

5) Statistical analysis

After completing the trial, a database was established using the Epidata 3.02 software [8], and all data were double entered; we used the SPSS 12.0 software for statistical analysis. The following items were analyzed: a) within each treatment group, the function evaluation results before and after treatment were compared using the paired t-test; b) the paired t-test was used to determine the functional differences after treatment between the experimental group and the control group.

\section{RESULTS}

The statistical analysis on the four functional assessment items of the experimental group indicated that the differences before and after treatment were highly significant $(P<0.01)$. However, in the control group statistical analysis on the four function assessment items showed that only the differences in EMG results before and after treatment were statistically significant $(P<0.05)$, while the difference of the other three assessment items were nonsignificant $(P>0.05)$. Between the two groups, the differences of all assessment items before treatment were nonsignificant, while the differences of all assessment items after treatment were highly significant $(P<0.01)$. The results are shown in Table 1.

\section{CONCLUSION}

The six-step method of the Chinese Daoyin technique is an effective method to rehabilitate foot drop in hemiparetic patients.

\section{DISCUSSION}

The treatment of motor function disorders due to cellular necrosis of the CNS must focus on the functional restoration of CNS neurons. Because CNS neurons are unable to regenerate, they have to be substituted by training the surrounding healthy neurons, and by activating the backup conduction pathways, which is a process of nervous 
Table 1. Functional assessment within and between groups before and after treatment.

\begin{tabular}{|c|c|c|c|c|c|}
\hline Assessment Item & Group & $\begin{array}{l}\text { Before Treatment } \\
\qquad(\bar{x} \pm s)\end{array}$ & $\begin{array}{l}\text { After Treatment } \\
\qquad(\bar{x} \pm s)\end{array}$ & $\begin{array}{l}P \text { Value within } \\
\text { Group }\end{array}$ & $\begin{array}{c}P \text { Value between } \\
\text { Groups }\end{array}$ \\
\hline \multirow[b]{2}{*}{ Muscle Strength } & six-step group & $1.32 \pm 0.65$ & $3.62 \pm 1.21$ & $<0.01$ & \multirow[b]{2}{*}{$<0.01$} \\
\hline & control group & $1.31 \pm 0.47$ & $2.13 \pm 1.01$ & $>0.05$ & \\
\hline \multirow{2}{*}{$\begin{array}{l}\text { EMG of Anterior Tibia } \\
\qquad(\mu \mathrm{V})\end{array}$} & six-step group & $26.18 \pm 8.36$ & $87.31 \pm 23.66$ & $<0.001$ & \multirow{2}{*}{$<0.05$} \\
\hline & control group & $27.32 \pm 10.54$ & $43.18 \pm 8.36$ & $<0.05$ & \\
\hline \multirow{2}{*}{$\begin{array}{l}\text { AROM of Ankle } \\
\text { Joint }\left(\left(^{\circ}\right)\right.\end{array}$} & six-step group & $9.23 \pm 2.36$ & $18.43 \pm 2.68$ & $<0.001$ & \multirow{2}{*}{$<0.01$} \\
\hline & control group & $10.12 \pm 2.36$ & $13.47 \pm 4.36$ & $>0.05$ & \\
\hline \multirow{2}{*}{$\begin{array}{c}\text { Fugl-Meyer } \\
\text { Assessment Score }\end{array}$} & six-step group & $0.43 \pm 0.50$ & $1.7 \pm 0.47$ & $<0.001$ & \multirow{2}{*}{$<0.01$} \\
\hline & control group & $0.50 \pm 0.50$ & $0.7 \pm 0.47$ & $>0.05$ & \\
\hline
\end{tabular}

potential exploitation. The human CNS has a tremendous amount of potential, the source of which is the massive quantity of backup cells and synapses, which is the foundation of the restoration of motor function after CNS damage. Therefore, the exploitation of nervous potential is the key to motor function restoration after CNS damage.

The CNS potential exploitation promotes plasticity and functional reorganization of the CNS. The plasticity of the CNS is based on the "skillful usage-reliability" principle [9], which states that the plasticity changes of the cortex motor center can only be achieved by actively using the muscles, and the lost motor function of the body can only be recovered when the functional reorganization of the CNS has been completed.

Traditional rehabilitation techniques such as those described by Bobath, Rood and Brunnstrom consist mainly of passive movement, primitive reflexes, and afferent stimulation, using lower central reflexes such as the stretch reflex and the tonic neck reflex to induce spontaneous movement of the body. Therefore, they are inconsistent with the "skillful usage-reliability" principle, which is essential for enhancing CNS plasticity changes and functional reorganization. This may be the reason why the effects of rehabilitation training using these methods are somewhat unsatisfactory [9]. For example, the cause of foot drop of the hemiparetic lower limb in stroke patients is that the motor center or conduction pathways of the opposite side of the brain are damaged. The brain loses the ability to control voluntary movement of the anterior tibial muscle, causing its paralysis, and finally resulting in foot drop. The voluntary movement of the anterior tibial muscle can only be restored after the function of the corresponding motor center and conduction pathways are restored. Only then can the function of foot dorsiflexion be regained. Because the reflex center for the Brunnstrom dorsiflexion facilitation methods is located in the spinal cord, the influence of this method on the motor center in the cerebral cortex is very limited. Therefore, it is inefficient in facilitating the plasticity and functional reorganization of the CNS, and the reason why the outcome of the control group is unsatisfactory [10].

The Chinese Daoyin technique can greatly arouse a patient's perseverance and determination to complete a certain action to achieve functional rehabilitation. This method has progressed significantly in history. For stroke rehabilitation, over twenty different Daoyin exercises were documented in the first volume of the "General Treatise on the Causes and Symptoms of Diseases" [1], which was written in the Sui dynasty (610 A.D.). This includes treatment methods for hemiparesis, limb spasms, and various other sequela of stroke. It contains static exercises in which the mind guides breathing, and dynamic exercises, which include movement (including imitation of animals). Daoyin exercises can dredge the channels, regulate qi and blood, coordinate zang (organ) and fu (viscera), and strengthen healthy qi to eliminate pathogenic factors by controlling the mind, breathing, and the body. Overall, it can increase the homeostatic state of the body and benefit metabolism. Modern medicine has proven that the Chinese Daoyin technique has good regulation and rehabilitation effects on cardiovascular, respiratory, neurological and digestive diseases [2]. Throughout history, researchers have supplemented and developed its many aspects. Many contents of it deserve studying and generalizing for the present day [3]. It has intimate connections and similarities with modern psychotherapy, athletic therapy, naturopathy and information therapy. It can be considered an integration of the above therapies. However, since the day of its creation and in the path of its development history, it has been supplemented, and modified by various denominations, and it has an inseparable relationship with traditional Chinese culture. Therefore, although its theories are very extensive and profound, it inevitably has a mysterious tone, 
causing it to lack a precise concept, and have subjective and abstract principles. Therefore, since the Ming and Qing Dynasty, it has been mainly used in the field of health care instead of clinical rehabilitation, and nowadays is near the point of extinction.

As we understand it, to control the body by adjusting the mind and breathing using the Chinese Daoyin technique is a process of guiding the patient to concentrate on completing a certain action. This process is consistent with the "skillful usage-reliability" principle to enhance remodeling of the CNS. Therefore, it facilitates functional reorganization of the CNS, and helps patients restore motor function more easily. However, the traditional Chinese Daoyin technique consists mainly of whole-body movements, such as "celestial being showing the way" and animal imitation, which are contrary to the rehabilitation principles of "step by step" and "from easy to difficult". Patients are unable to complete the movements due to their difficulty, and repeated failure will lead to loss of confidence for rehabilitation.

The six-step method of the Chinese Daoyin technique has been improved by combining modern medical theories and the basic principles of the traditional Daoyin technique. The functional training after damage to the CNS should emphasize on the restoration of voluntary movement. To perform a highly complex and purposeful task, the programming of the motor program and the onset of motion is closely related to the motor association area of the brain. This function is destroyed to various degrees when the motor center of the cerebral cortex is damaged and needs to be reestablished to restore motor function. When initiating a specific voluntary movement, the patient needs to judge motion target and body position, determine the manner, timing and action speed, and then reach the borderline state of motion. Therefore, the first step of the six-step Daoyin method is motion imagination and imitation. The purpose of this step is to help the patient form the idea of establishing voluntary movement, find the basic motor pattern and the final goal, and use mind and breathing control to direct the patient to the borderline state of motion. In addition, while ensuring that there is only one single target muscle, the therapist guides the patient to fire motor signals "abruptly" to the target muscle, which can localize the point of exertion and results in better effects. The purpose of counting while resisting the applied force is to quantify the amount of training while guiding the patient's brain to fire signals at the correct target, which exploits the CNS potential of the patient more effectively. In the meantime, encouraging the patient and using phrases such as "stand up or you'll fall", "lift or you'll trip" to create a "critical situation" to urge the patient to com- plete the appointed task to the extent of his/her abilities. The entire process of the six steps is an attempt to result in better exploitation of the CNS and functional reorganization.

Because the electric signals released from the brain cells to drive and control muscles are a form of energy, the six-step method is a process of using the internal energy flow of the body to facilitate plasticity changes and functional reorganization of the CNS.

The Chinese Daoyin technique controls the body by controlling the mind and breathing, it is a good method to exploit CNS potential, facilitate functional reorganization, and eventually restore independent innervations of paralyzed muscles. Its modern interpretations and development can increase the effect of rehabilitation and better display ancient Chinese rehabilitation techniques and their contributions to the hemiparetic patient.

\section{REFERENCES}

[1] Ding, G.D. (1993) General treatise on the causes and symptoms of diseases. People's Medical Publishing House, Beijing, 1-2. (In Chinese).

[2] Chen, K.Y. (1988) Chinese traditional rehabilitation medicine. People's Medical Publishing House, Beijing, 170-171. (In Chinese).

[3] Cao, H.H. (2003) Is Daoyin a kind of gymnastics? Journal of Beijing University of Physical Education, 26, 859. (In Chinese).

[4] Hong, D. (2004) Rehabilitation medicine. Peking University Medical Press, Beijing, 26. (In Chinese).

[5] Posner, M.I. and Raichle, M.E. (1998) The neuroimaging of human brain function. Proceedings of the National Academy of Sciences of the USA, 95, 763-764. doi:10.1073/pnas.95.3.763

[6] Lv, H.Y. (2007) Research on traditional Chinese medicine basic theories combined with molecular biology. Chinese Archives of Traditional Chinese Medicine, 9, 1909-1910. (In Chinese).

[7] Miu, H.S. (2000) Rehabilitation medicine: Principles and practice (Volume 1). Shanghai Scientific \& Technical Publishers, Shanghai, 651. (In Chinese).

[8] Li, Y.M., Feng, Z.C., Li, X.K., et al. (2010) Application of Epidata and SPSS in statistical entry and analysis of the multiple-choice data. Modern Preventive Medicine, 37, 4012-4014.

[9] Zhu, Y.L. (2005) Plasticity of the brain and neurorehabilitation. Chinese Journal of Neurology, 38, 591-592. (In Chinese).

[10] Zhang, L.Y. (2004) Research progress and application of treatment technique for stroke rehabilitation. Chinese Journal of Clinical Rehabilitation, 8, 7768-7769. (In Chinese). 